ROSs, M. D., AND SHAW, R. F. 1971. Maintenance of male sterility in plant populations. Heredity, 26, 1-8.

SHAW, R. F. 1957. The theoretical genetics of the sex ratio. Genetics, 43, 149-163.

WEIR, B. s., ALLARD, R. W., AND KAHLER, A. L. 1972. Analysis of complex allozyme polymorphisms in a barley population. Genetics, 72, 505-523.

\title{
GIEMSA STAINING AND THE DISTRIBUTION OF HETEROCHROMATIN IN RYE CHROMOSOMES
}

\author{
S. C. VERMA and H. REES \\ Department of Agricultural Botany, University College of Wales, Aberystwyth
}

Received 12.ix.73

\section{SUMMARY}

$C$-banding, by Giemsa staining, is largely restricted to distal regions of rye chromosomes. This applies, also, to B chromosomes. The distribution of C-bands coincides with that of distally localised heterochromatin. The area of metaphase chromosomes staining with Giemsa corresponds to the area occupied by heterochromatin in interphase nuclei as revealed by Feulgen staining. The Giemsa-stained $C$-bands account, therefore, for all the heterochromatin in rye nuclei. Individual chromosomes within the complement are readily identified following Giemsa staining.

\section{INTRODUCTION}

Giemsa-staining methods developed, initially, by Pardue and Gall (1970) have revealed hitherto undetectable and indeed unsuspected patterns of structural differentiation in metaphase chromosomes. The following account describes how Giemsa staining in rye may be used to identify individual chromosomes within the complement and, as well, to map the distribution of heterochromatin within the chromosomes.

\section{Materials AND MEthods}

The rye plants used were from a population carrying $B$ chromosomes, the $\mathrm{B}$ frequency ranging from 0 to 8 per plant. The population was derived initially from crosses between plants of Secale cereale, of the variety Stälrag, and S. vavilovii plants carrying B's (see Jones and Rees, 1967). We could detect no morphological difference between the chromosome complement of this population and those of "pure" $S$. cereale populations. The reason for choosing this particular population was that it contained many B's.

Seeds were germinated on moist filter paper in petri dishes in the dark at $20^{\circ}$ C. Roots from seedlings with three to five roots were immersed in 0.2 per cent colchicine for 5 hours and fixed, subsequently, for 4 hours in 1:3 acetic acid : absolute alcohol. The Giemsa-staining procedure used was that devised by Vosa and Marchi (1972) for plant material. The technique was as follows:

1. Hydrolysis in $1 \mathrm{~N} \mathrm{HCl}$ at $60^{\circ} \mathrm{C}$. for 25 seconds, followed by thorough washing in distilled water. Further softening of root tips by immersion in 45 per cent acetic acid. 
2. Root tips squashed in 45 per cent acetic acid on albuminised coverslips. Coverslips removed by inverting over absolute alcohol.

3. Coverslips into a saturated aqueous solution of Barium hydroxide for 5 minutes, followed by thorough rinsing in running distilled water.

4. Incubation at $60^{\circ} \mathrm{C}$. in $2 \times \mathrm{SSC}$ (salt-sodium citrate) solution for 1 hour. Rinsed in distilled water.

5. Coverslips in "improved" Giemsa R66 (by Gurr) at $p \mathrm{H} 6.8$ for 5-10 minutes. Rinsed in distilled water.

6. Coverslips mounted in Canada Balsam after dipping three times in xylene.

The darkly stained bands ( $C$-bands) which show up in metaphase chromosomes by this method are said to correspond with the location of constitutive heterochromatin (Cooper and Hsu, 1972; Hsu and Arrighi, 1972; Schweizer, 1973).

The amount of heterochromatin in interphase nuclei was estimated as follows. First, root tips were stained by the standard Feulgen method. Second, using the "scanning dot" of an M85 Vickers microdensitometer, the optical density (O.D.) of the heterochromatin was determined. For this purpose spot densities measured in five heterochromatic blobs were averaged to give the O.D. for the heterochromatin in each nucleus. Third, the nucleus was scanned at the O.D. of the heterochromatin to give the area occupied by heterochromatin. Finally, the nucleus was scanned at an O.D. of 0 to obtain the total area of the nucleus. The area of heterochromatin was then expressed as a percentage of the total. The area of heterochromatin was calculated for each of 10 nuclei in root tips from two plants.

\section{Results}

\section{(i) The distribution of $C$-bands}

Fig. 1 and plate I show, (a) $C$-bands at the distal ends of all short arms, although the narrow band in the short arm of chromosome 1 is sometimes difficult to detect and, occasionally, undetectable. (b) $C$-bands at the distal ends of long arms in five of the seven chromosomes. (c) A narrow band proximal to the secondary construction in chromosome 7. (d) a $C$-band at the end of the long arm of B chromosomes.

\section{(ii) Identification}

On the basis of total length and of arm ratio the classification of the rye complement at metaphase of mitosis is extremely difficult (Sybenga and Wolters, 1972). For one thing the lengths of chromosomes relative to one another vary substantially between cells. The same is true for arm ratios. Also, as Sybenga and Wolters point out, there is often a considerable variation in length and arm ratio even between homologous chromosomes within the same cell. For this reason a reliable "karyotype" of mitotic chromosomes in rye can be constructed only by measurements in large samples of metaphases. The relative lengths and arm ratios in fig. 1 are, indeed, based on such measurements. Measurements in our own material do not differ significantly from those by Sybenga and Wolters.

Fig. 1 also shows, to scale, the lengths and distribution of $C$-bands in the seven chromosomes of the normal complement and in the $\mathrm{B}$ chromosome. 
The banding pattern, in itself, is an extremely useful means for identifying the chromosomes. In conjunction with the arm ratios and relative lengths they are a very considerable asset indeed. A simple key to identification is depicted in table 1 .

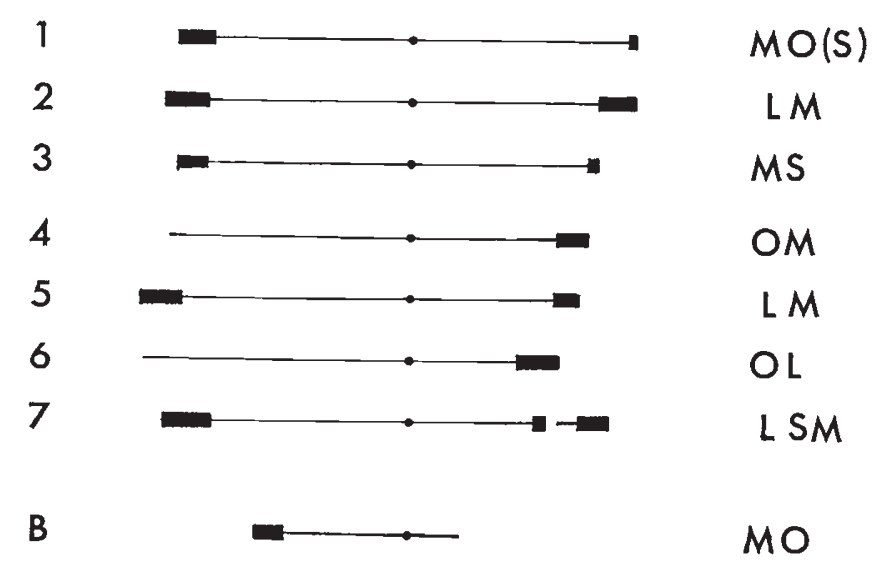

FIG. 1.-The distribution and extent of $C$-bands in the rye complement, and in a B chromosome. Although chromosome 1 is in more than 90 per cent of cells $M O$, i.e. without a $C$-band in the short arm, the extent of the $C$-band occasionally appearing in that arm is shown in the figure.

There is, of course, some variation in the degree of staining and consequently in the width of bands. For example in many cells chromosome 5 may be classified as $M M$ rather than $L M$ as given in the key, where the maximum band widths are represented (as is the case, also, in fig. 1). Despite this variation the identification of individual chromosomes is relatively easy.

\section{TABLE 1}

The location and extent of $\mathrm{C}$-bands in the rye complement. The numbering of chromosomes follows that of Sybenga and Wolters (1972)

\begin{tabular}{lccc} 
& \multicolumn{2}{c}{ C-banding } & \\
\cline { 2 - 3 } "Metacentrics" & \multicolumn{2}{c}{ Chromosome } \\
& $M$ & $O(S)$ & 1 \\
& $L$ & $M$ & 2 \\
& $M$ & $S$ & 3 \\
& $O$ & $M$ & 4 \\
"Sub-metacentrics" & $L$ & $M$ & 5 \\
Nucleolar chromosome & $O$ & $L$ & 6 \\
& $L$ & $S M$ & 7
\end{tabular}

\section{(iii) Heterochromatin}

Table 2 gives, as percentages of the total, the areas of interphase nuclei occupied by the heterochromatin. The areas were estimated as described under Material and Methods.

In well-stained metaphases (with $2 \mathrm{~B}$ 's) the $C$-bands occupy 14 per cent 
of the total chromosome area. This falls within the range of estimates for the area occupied by heterochromatin in interphase. It is safe to conclude therefore that the $C$-bands at metaphase correspond, as expected, to the heterochromatin as revealed in interphase. They account moreover for all such heterochromatin.

\section{TABLE 2}

Heterochromatin, as percentage of the total area, of each of 10 interphase nuclei in two plants, both with $2 B$ chromosomes

\begin{tabular}{ccc} 
& Plant 1 & Plant 2 \\
1 & 12.53 & 11.33 \\
2 & 12.84 & 12.53 \\
3 & 15.98 & 13.35 \\
4 & 11.55 & 11.94 \\
5 & 13.88 & 13.42 \\
6 & 11.75 & 12.27 \\
7 & 15.51 & 12.39 \\
8 & 15.34 & 12.34 \\
9 & 11.99 & 12.85 \\
10 & 11.95 & \multicolumn{2}{c}{13.95} \\
Mean & 13.33 & \multicolumn{2}{c}{12.64}
\end{tabular}

The distribution of $C$-bands at the ends of all short arms and five of the seven long arms (fig. 1) corresponds precisely with the distribution of heterochromatic knobs as revealed by the rye pachytene analysis of Lima de Faria (1952). There is, however, no $C$-banding in the vicinity of the centromeres to correspond with the densely compacted segments in these regions at pachytene. We conclude, therefore, that these segments are not, in the strict sense, heterochromatic as described by Lima de Faria (loc. cit. cf. Darlington and Haque, 1966; Ayonoadu and Rees, 1973). In this connection it is necessary to point out that the Giemsa-staining pattern at pachytene corresponds with that at mitotic metaphase. The terminal knobs are densely stained with Giemsa whereas there is no trace of stain around the centromeres.

\section{REFERENCES}

AyonoAdu, U. W., AND REEs, H. 1973. DNA synthesis in rye chromosomes. Heredity, 30, 233-240.

COOPER, J. E. K., AND HSU, T. C. 1972. The C-band and G-band patterns of Microtus agrestis chromosomes. Cytogenics, 11, 295-304.

DARLINGTON, C. D., AND HAQUE, A. 1966. Organisation of DNA synthesis in rye chromosomes. Chromosomes Today, 1, 102-107.

HSU, T. C., AND ARRIGHI, E. 1972. Comments on the fluorochrome and heterochromatin staining techniques. In Perspectives in Cytogenetics. The next decade, Eds. S. W. Wright et al., 197-200. Publ. Charles C. Thomas, Springfield, U.S.A.

JONES, R. N., AND REES, H. 1967. Genotypic control of chromosome behaviour in rye. XI. The influence of $B$ chromosomes on meiosis. Heredity, 22, 333-347.

LIMA DE FARIA, A. 1952. Chromomere analysis of the chromosome complement of rye. Chromosoma, 5, 1-68.

PARDUE, M. L., AND GALl, J. G. 1970. Chromosomal localization of mouse satellite DNA. Science, 168, 1356-1358. 


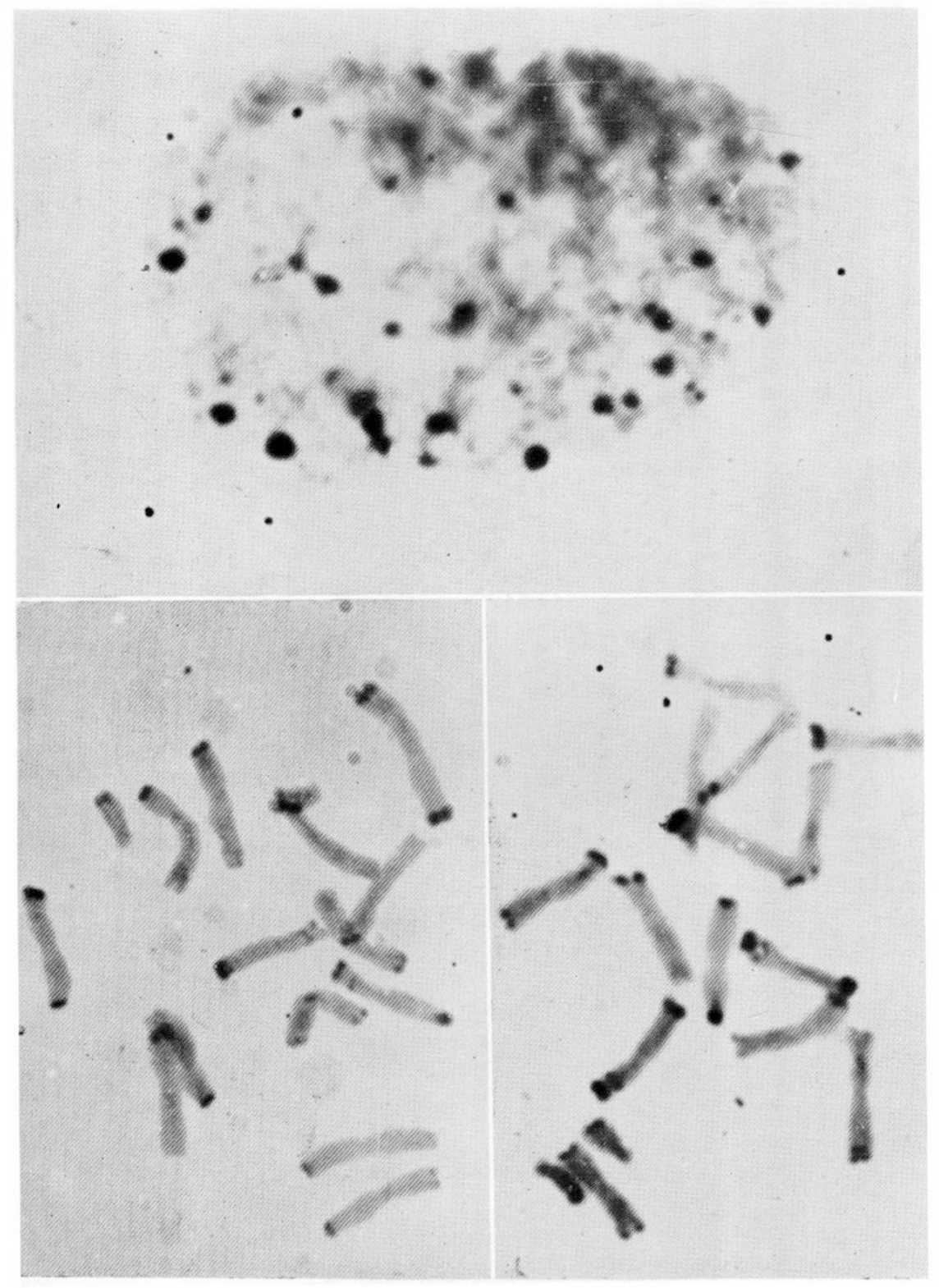

Plate I

a, Heterochromatin at interphase from a rye root-tip meristem. $b, c, C$-banding at metaphase of mitosis. 
SCHWEIzER, D. 1973. Differential staining of plant chromosomes with Giemsa. Chromosoma (Berl.), 40, 307-320.

SYBENGA, J., AND WOLTERS, A. H. G. 1972. The classification of the chromosomes of rye (Secale cereale L.): A translocation tester set. Genetica, 43, 453-464.

VOSA, C. G., AND MARCHI, P. 1972. Quinacrine fluorescence and Giemsa staining in plants. Nature New Biology, 237, 191-192.

\title{
MULTI-LOCUS POLYMORPHISM AND SELECTION IN A POPULATION OF DROSOPHILA MELANOGASTER
}

\section{LINKAGE DISEQUILIBRIUM ON CHROMOSOME III}

\author{
A. J. BIRLEY \\ Department of Genetics, University of Birmingham, P.O. Box 363, \\ Birmingham B15 2TT, England
}

Received 29.ix.73

\section{Summary}

Linkage disequilibrium was detected between three polymorphic loci (octanol dehydrogenase, esterase- 6 and pink) on 3rd chromosomes extracted from a population of Drosophila melanogaster. Over the chromosome segment of 12.4 centimorgans, the alleles at all three loci were not associated at random. Evidence is presented suggesting that the observed linkage disequilibria are maintained by selection.

\section{INTRODUGTION}

AT least 30 per cent of the electrophoretically detected proteins within populations of many species are polymorphic (Harris, 1966, 1969; Hubby and Lewontin, 1966; Lewontin and Hubby, 1966; Selander and Yang, 1969; Ayala and Powell, 1972). As yet the organisation of this wealth of genetic variation within the genome has received little attention from experimental population geneticists. Studies in theoretical population genetics show that a great deal of linkage disequilibrium may be the norm within populations; selection generally acts upon groups of highly correlated genes rather than upon individual loci (Franklin and Lewontin, 1970). For populations of outbreeding organisms only small amounts of linkage disequilibrium have been reported between the alleles at individual loci. In populations of Drosophila melanogaster, Mukai et al. (1971) did not find any linkage disequilibrium between three protein loci on chromosome II, and Charlesworth and Charlesworth (1971) report that only four out of 30 tests for non-random association between alleles at loci on chromosome III were statistically significant. In contrast, there is striking evidence for selectively maintained linkage disequilibria in populations of the inbreeding cereals, Hordeum vulgare (Glegg, Allard and Kahler, 1972) and Avena barbata (Allard, Babbel, Clegg and Kahler, 1972).

In the present study, linkage disequilibrium was studied between three polymorphic loci on chromosome III in a long established population of D. melanogaster. 